\section{IUCrJ}

ISSN 2052-2525

BIOLOGY|MEDICINE

\title{
Mirolysin structures open a window on gum disease
}

\author{
Evette S. Radisky*
}

Department of Cancer Biology, Mayo Clinic, Jacksonville, Florida 32224, USA. *Correspondence e-mail: radisky.evette@mayo.edu

Keywords: periodontitis; peptidase; protease; crystal structure; zymogen activation; substrate specificity.

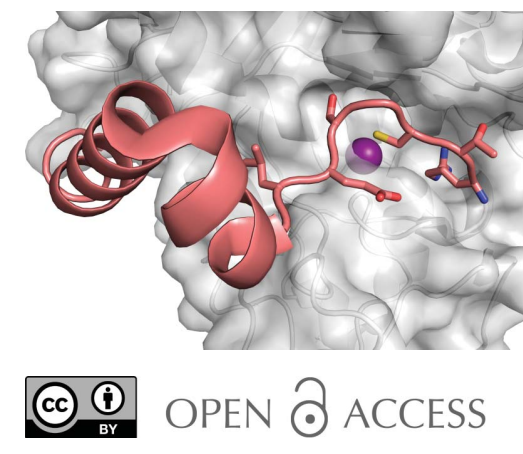

Periodontitis, commonly known as gum disease, is a chronic inflammatory disease characterized by dysregulation of the oral microbiota in the subgingival space below the gum line (Hajishengallis, 2015). Healthy gums are compromised by a proliferation of pathogenic anaerobic bacteria, most commonly the triad of Porphyromonas gingivalis, Treponema denticola and Tannerella forsythia. These pathogens release 'virulence factors', molecules that aid their colonization and survival in the subgingival niche and their evasion of the host immune response. Synergistic interactions of the pathogenic microbes with normal oral microbiota lead to a sustained host inflammatory response, degrading periodontal tissues, eroding bone, and ultimately leading to tooth loss. Severe periodontitis affects around $10 \%$ of the human population, and health implications are not limited to oral health; the chronic inflammatory conditions precipitated by the disease can extend to systemic manifestations, contributing to risk of cardiovascular and respiratory diseases, diabetes, rheumatoid arthritis, and cancer (Hajishengallis, 2015; Peters et al., 2017).

Proteases are amongst the most abundant virulence factors produced by periodontal pathogens. These enzymes degrade host proteins to feed bacterial growth, and also serve as key mediators of immune-subversive mechanisms, for example by degrading or inactivating endogenous antimicrobial peptides and components of the host complement system (Hajishengallis, 2015). One of the proteases implicated in these activities is mirolysin, a metalloprotease secreted by $T$. forsythia. Mirolysin cleaves multiple components of the complement pathway to protect the pathogen against complementmediated killing, and degrades the antimicrobial peptide LL-37, a human host defense peptide secreted by epithelial and immune cells at sites of infection (Jusko et al., 2015; Koneru et al., 2017). As a recently discovered mediator of $T$. forsythia survival and immune evasion, mirolysin offers a potential therapeutic target, yet until now the structural basis for its latency, activation and substrate specificity have not been defined.

In this issue of IUCrJ, Guevara, Rodriguez-Banqueri et al. report high-resolution crystal structures of promirolysin, defining the mechanism of latency of the precursor protein (Guevara et al., 2020). Like nearly all proteases, mirolysin is first produced as a zymogen, maintained in an inactive state by the presence of an N-terminal extension known as a pro-peptide, pro-segment or pro-domain (Fig. 1). Upon proteolytic cleavage of the pro-segment, the active site of a protease becomes exposed and accessible to substrates. While unified by this common theme, different clans and families of metalloproteases show myriad variations in the details of how latency is maintained and activation subsequently achieved. These variations have been carefully cataloged in over 60 crystal structures of metalloprotease zymogens, including many from the Gomis-Rüth research group (Arolas et al., 2018). Here, the group finds that the mechanism of latency of promirolysin closely resembles the cysteine switch mechanism of matrix metalloproteinases (MMPs) and a number of other zinc metallopeptidases (Morgunova et al., 1999; Arolas et al., 2018). A stretch of the pro-segment occupies the enzyme active site cleft in an orientation opposite to that of a productively bound substrate, positioning a conserved cysteine residue to bind the catalytic zinc. The cysteine side chain displaces the normally bound water molecule that plays the essential hydrolytic role in proteolysis, thus rendering the zymogen inactive.

While this basic cysteine switch mechanism of latency is well precedented, the promirolysin structure reveals a surprisingly extensive interface between the prosegment and the catalytic domain. The zymogen structure reveals a buried surface area of $2302 \AA^{2}$ between these structural elements, greater than the average buried surface area 


\section{scientific commentaries}

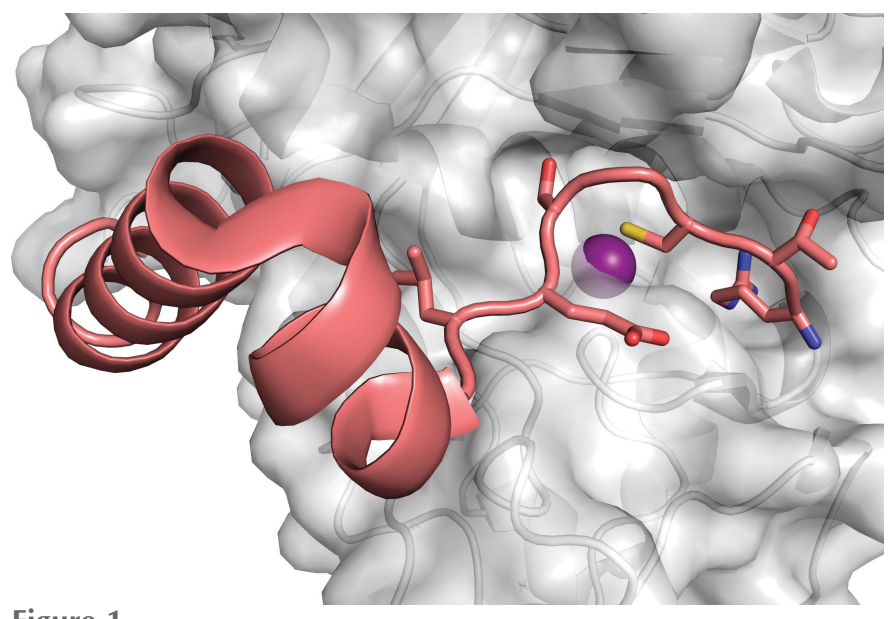

Figure 1

Structure of promirolysin reveals how the pro-segment inhibits enzyme activity prior to proteolytic activation. The N-terminal residues of the pro-segment (salmon) occupy the active site cleft of the catalytic domain (gray), with a key cysteine residue coordinating the catalytic zinc ion (purple). This figure was generated using Pymol from the coordinates of PDB structure $6 r 7 v$, reported in this issue by Guevara, RodriguezBanqueri et al.

for protein-protein complexes, which is remarkable given the small size of the 34-residue pro-segment. The pro-segment and catalytic domain interact through a balance of favorable hydrophobic interactions complemented by an array of hydrogen bonds and salt bridges, the same forces that shape affinity and selectivity of intermolecular protein-protein interactions (Xu et al., 1997). The extensive nature of the broad interface between pro-segment and catalytic domain suggests the possibility that the pro-segment may be capable of inhibiting the enzyme in trans, and serving as a template for engineering protein-based mirolysin inhibitors of therapeutic utility. Similar approaches have shown promise for engineering therapeutic inhibitors of ADAM family metallopeptidases, based upon their much larger pro-domains (Moss et al., 2007; Wong et al., 2016).

Guevara, Rodriguez-Banqueri et al. further report a highresolution structure of active mirolysin bound to a peptide product, providing insight into determinants of substrate specificity of the protease (Guevara et al., 2020). A preference of mirolysin for cleavage on the $\mathrm{N}$-terminal side of basic residues (Koneru et al., 2017) is explained by the presence of an aspartate residue positioned to form a salt bridge with the substrate. This aspartate residue is conserved among the pappalysin family of metalloproteases to which mirolysin belongs, suggesting that similar specificity may be typical of the entire family, and that the mirolysin structure can offer a model to understand substrate recognition for the larger family. Additionally, this serendipitously trapped product complex may provide yet another starting point from which active site-targeted mirolysin inhibitors might be developed. A similar serendipitous discovery of a product fragment captured in a crystal structure of MMP-13 recently led to the design of a potent peptidomimetic inhibitor of this metalloprotease (Gall et al., 2019). Together the new structures of mirolysin reported here offer a window from which to understand the function of this protease in gum disease, with a glimpse of how one might inhibit this intriguing target.

\section{References}

Arolas, J. L., Goulas, T., Cuppari, A. \& Gomis-Rüth, F. X. (2018). Chem. Rev. 118, 5581-5597.

Gall, F. M., Hohl, D., Frasson, D., Wermelinger, T., Mittl, P. R. E., Sievers, M. \& Riedl, R. (2019). Angew. Chem. Int. Ed. 58, 40514055.

Guevara, T., Rodriguez-Banqueri, A., Ksiazek, M., Potempa, J. \& Gomis-Ruth, F. X. (2020). IUCrJ, 7, 18-29.

Hajishengallis, G. (2015). Nat. Rev. Immunol. 15, 30-44.

Jusko, M., Potempa, J., Mizgalska, D., Bielecka, E., Ksiazek, M., Riesbeck, K., Garred, P., Eick, S. \& Blom, A. M. (2015). J. Immunol. 195, 2231-2240.

Koneru, L., Ksiazek, M., Waligorska, I., Straczek, A., Lukasik, M., Madej, M., Thøgersen, I. B., Enghild, J. J. \& Potempa, J. (2017). Biol. Chem. 398, 395-409.

Morgunova, E., Tuuttila, A., Bergmann, U., Isupov, M., Lindqvist, Y., Schneider, G. \& Tryggvason, K. (1999). Science, 284, 1667-1670.

Moss, M. L., Bomar, M., Liu, Q., Sage, H., Dempsey, P., Lenhart, P. M., Gillispie, P. A., Stoeck, A., Wildeboer, D., Bartsch, J. W., Palmisano, R. \& Zhou, P. (2007). J. Biol. Chem. 282, 35712-35721.

Peters, B. A., Wu, J., Pei, Z., Yang, L., Purdue, M. P., Freedman, N. D., Jacobs, E. J., Gapstur, S. M., Hayes, R. B. \& Ahn, J. (2017). Cancer Res. 77, 6777-6787.

Wong, E., Cohen, T., Romi, E., Levin, M., Peleg, Y., Arad, U., Yaron, A., Milla, M. E. \& Sagi, I. (2016). Sci. Rep. 6, 35598.

Xu, D., Tsai, C. J. \& Nussinov, R. (1997). Protein Eng. 10, 999-1012. 